Rabaska

Revue d'ethnologie de l'Amérique française

BLANCHETTE, JEAN-FRANÇOIS. Du coq à l'âme. L'art populaire

au Québec. Ottawa, Presses de l'Université d'Ottawa, et Gatineau, Musée canadien de l'histoire, collection " Mercure ", 2014, 322 p. ISBN 978-2-7603-0814-5

\title{
Pascale Galipeau
}

Volume 12, 2014

URI : https://id.erudit.org/iderudit/1026804ar

DOI : https://doi.org/10.7202/1026804ar

Aller au sommaire du numéro

Éditeur(s)

Société québécoise d'ethnologie

ISSN

1703-7433 (imprimé)

1916-7350 (numérique)

Découvrir la revue

Citer ce compte rendu

Galipeau, P. (2014). Compte rendu de [BLANCHETTE, JEAN-FRANÇOIs. Du coq à l'âme. L'art populaire au Québec. Ottawa, Presses de l'Université d'Ottawa, et Gatineau, Musée canadien de l'histoire, collection " Mercure ", 2014, 322 p.

ISBN 978-2-7603-0814-5]. Rabaska, 12, 245-247.

https://doi.org/10.7202/1026804ar d'utilisation que vous pouvez consulter en ligne. 
et généalogique. Espérons qu'une version numérique pourra compenser cette lacune dans un avenir prochain.

\section{MARCEL Fournier}

Longueuil (Québec)

Blanchette, Jean-François. Du coq à l'âme. L'art populaire au Québec. Ottawa, Presses de l'Université d'Ottawa, et Gatineau, Musée canadien de l'histoire, collection «Mercure », 2014, 322 p. ISBN 978-2-7603-0814-5.

Dans la foulée de l'exposition $D u$ coq à l'âme présentée au Musée canadien des civilisations en 2008, Jean-François Blanchette offre une somme généreuse des collections du Musée canadien de l'histoire, doublée d'un regard critique sur le développement de l'art populaire au Québec. L'ouvrage est savant, mais sans affectation. Blanchette écrit au « je », raconte ses bons et ses mauvais coups comme il dévoile ses stratégies d'enquête sans gêne. Il privilégie le récit de vie qui permet des questions ouvertes et débouche parfois sur des confidences. Sa sensibilité le met au diapason des artistes. «On l'a rarement mentionné, mais la créativité populaire naît aussi de la critique sociale, de la révolte, de l'angoisse, du désespoir et de toutes les misères de l'humanité qui alimentent le tourbillon de la vie. Car, comment peut-on vivre sans dormir, peindre sans pouvoir s'arrêter, gosser à s'en faire des ampoules, comme le fait Clémence Lessard, s'il n'y avait pas une motivation profonde qui brûle à l'intérieur de soi ? »

Un chapitre passionnant est consacré à Nettie Sharpe, cette collectionneuse dont la magnifique collection d'art populaire et d'antiquités québécoises fut acquise par le Musée en 2002. Anglophone parfaitement bilingue et résolument dédiée au patrimoine des Québécois francophones, elle a sauvé de l'oubli (ou du feu !) plusieurs milliers d'objets. Son entrevue permet de mieux connaître le parcours de la visionnaire infatigable qui s'est consacrée, 75 ans durant, à sa mission de rapailleuse. La section sur le mouvement de renaissance artisanale est tout aussi étoffée. Dans le contexte des grandes enquêtes sur les ressources naturelles, réalisées à la demande de Duplessis à la fin des années 1930, l'inventaire des ressources artisanales va donner l'occasion de prendre le pouls de la production susceptible d'intéresser le touriste. L'artisanat apparaît comme un filon salvateur en ces temps de crise.

Tout au long de son ouvrage, Blanchette a l'honnêteté de questionner sur la pratique, comme lorsqu'il fait un retour sur les dérives de l'interprétation dans le chapitre sur l'art traditionnel et ses clones. Dans un mea-culpa courageux, il raconte que plusieurs ethnologues, dont lui-même, croyaient que la tradition s'était maintenue malgré le modernisme. L'exemple des fameux 
modèles réduits qui évoquent les travaux traditionnels et qu'on appelait des mémoires en bois est réévalué. « Il s'avère que ces mémoires en bois sont un piège d'interprétation, car on ne les fabrique que depuis l'avènement de la modernité. Les raisons mêmes de leur fabrication en témoignent : ces traditions n'existent plus ». Blanchette fait également son autocritique lorsqu'il s'interroge sur les grandes lignes de force qui sous-tendaient l'exposition $D u$ fond $d u$ coeur $^{1}$ à laquelle il avait participé sous la direction de Pierre Crépeau. À l'époque, les objets avaient été regroupés sous diverses rubriques : Reflets, Allégeances, et Fantaisies. « Il semble bien, trente ans plus tard, que nous avons eu tort. La tendance n'a été que dans une seule direction : celle de la modernité. »

Le chercheur fait aussi preuve de hardiesse en incluant dans son panorama la production des artistes du graffiti. Tout comme il choisit la terminologie de l'art indiscipliné pour décrire la création contemporaine, « cette forme de créativité personnelle non influencée par la tradition. » Également, l'auteur n'a pas peur de déboulonner des statues lorsqu'il parle du mimétisme dans l'art populaire en citant le cas du folkloriste Jean-Claude Dupont qui s'inspire des artistes populaires sans l'être lui-même.

Le survol de l'art populaire est accompagné d'archives exceptionnelles (du Musée et d'ailleurs) qui avaient illuminé l'exposition. On retrouve avec bonheur les photos de Villeneuve, père et fils, à Manic V; des enfants photographiés par Marius Barbeau à Cap-aux-Oies en 1937 ; le sourire d'Yvonne Bourgault... et tous les contemporains avec leurs œuvres en abondance. Si le format du livre n'en fait pas un livre prestigieux (coupures oblige), il a le mérite d'être abondamment illustré et d'un prix abordable.

Mais, évidemment il y a toujours un « mais ». La deuxième partie du livre consacrée à la sélection des œuvres s'appuie sur de grandes catégories qui, pas plus que celles $D u$ fond $d u$ cœur $^{2}$, ne me semblent fondées. Je me questionne beaucoup sur ce besoin de classer, d'opposer, de sélectionner... et nous en avons là un bel exemple, avec cette liste des " valeurs identitaires » de l'art populaire québécois. Pour sa démonstration, Blanchette dégage une série de qualificatifs associés en paire, qui vont servir à regrouper les œuvres : Enraciné-Pluriel, Fidèle-Rebelle, Fier-Raconteur, et Jouisseur-Excessif. Pourquoi se lancer dans cette catégorisation ? Est-ce bien nécessaire pour apprécier les œuvres ? N'est-ce pas là une série de descriptifs universels ? Les Québécois seraient-ils seuls à y prétendre ? Pour faire passer son message, le chercheur cloisonne la réalité. Ce me semble maladroit d'essayer d'enfermer une création qui, par essence, n'entre pas dans la catégorie où on voudrait

1. Pierre Crépeau et al., Du fond du cœeur. L'art populaire au Canada, Ottawa, Musée national de l'homme, 1983.

2. Ibid. 
la restreindre. Les œuvres sont belles, les propos des artistes souvent très émouvants, mais c'est au niveau de l'interprétation que ça se corse. D'autres chercheurs sont tombés dans le même piège : on se rappellera que les auteurs de Pour passer le temps ${ }^{3}$ avaient, eux aussi, fixé un critère pour sélectionner leurs artistes, celui de la reconnaissance par les pairs. Ce qui écartait d'emblée tous les marginaux que la communauté ne voulait pas intégrer dans ses rangs. Du coq à l'âme bâtit des catégories de valeurs fourre-tout. A-t-on besoin de tout classifier pour apprécier les œuvres d'art?

On aura compris que cette critique n'est qu'un bémol devant le travail important livré par Blanchette. Ce dernier débroussaillage de l'art populaire québécois apporte un nouvel angle de connaissance et c'est la preuve de sa grande hardiesse.

Pascale Galipeau

Canton de Hatley

Boivin, AuréLien. Contes, légendes et récits de l'île de Montréal. Tome 2. Montréal : une ville imaginée. Notre-Dame-des-Neiges, Éditions TroisPistoles, 2013, 851 p. ISBN 978-2-89583-274-4.

Concentré autour d'un thème très évocateur, ce deuxième tome des Contes, légendes et récits de l'île de Montréal rassemblé par le professeur Aurélien Boivin favorise la mise en valeur d'un imaginaire souvent méconnu autour de cette métropole dont il est parfois difficile de croire qu'elle peut encore nous révéler quelque chose de neuf. En fait, Montréal revu par ses écrivains les plus connus et surtout par d'autres malheureusement trop ignorés révèle encore bien des facettes insoupçonnées pour le lecteur qui prend le temps de s'arrêter à cette somme inestimable si bellement imprimée par les Éditions Trois-Pistoles.

La présentation d'Aurélien Boivin, en début de volume, sait nous intéresser à la liste des textes qu'il a retenue. La division en trois sections peut apparaître générale et même un peu courte, mais elle sert bien le projet de relater un imaginaire dont la valeur apparait plus nettement dans cet ensemble littéraire vaste mais plein de recoupements possibles. Certains lecteurs préféreront l'un ou l'autre des regroupements : nouvelles policières, souvenirs, nouvelles réalistes. Convenons que cette brique n'est pas un livre qu'il faut lire d'un seul coup ; alors, pourquoi ne pas choisir selon ses préférences ? Je peux dire par expérience que l'on a souvent le goût d'y revenir et que cela fait

3. Jean Simard, Bernard Genest, Francine Labonté et René Bouchard, Pour passer le temps : artistes populaires du Québec, Québec, Ministère des Affaires culturelles, coll. " les Cahiers du patrimoine $», \mathrm{n}^{\circ} 17,1985$. 